\title{
Use of CT Angiography and Digital Subtraction Angiography in Patients with Ruptured Cerebral Aneurysm: Evaluation of a Large Multihospital Data Base
}

\author{
J.S. McDonald, D.F. Kallmes, G. Lanzino, and H.J. Cloft
}

\begin{abstract}
BACKGROUND AND PURPOSE: Both CT angiography and digital subtraction angiography are used to detect aneurysms in patients with subarachnoid hemorrhage. We examined a large multihospital data base to determine how practice is evolving with regard to the use of CT angiography and DSA in patients with ruptured cerebral aneurysm.
\end{abstract}

MATERIALS AND METHODS: The Premier Perspective data base was used to identify hospitalizations of patients treated with clipping or coiling of ruptured cerebral aneurysms from 2006-2011. Billing information was used to determine pretreatment and posttreatment use of DSA and CT angiography during hospitalization.

RESULTS: A total of 4972 patients (1022 clipping, 3950 coiling) at 116 hospitals were identified. The percentage of patients with SAH who underwent pretreatment CT angiography significantly increased from 20\% in 2006 to $44 \%$ in $2011(P<.0001)$, whereas the percentage of patients who underwent DSA remained unchanged from $96-94 \%(P=.28)$. This CT angiography trend was observed in coiling patients $(17-42 \%, P<.0001)$ and clipping patients $(32-54 \%, P<.0001)$. There was a significant increase in the percentage of patients who underwent posttreatment imaging from $41 \%$ in 2006 to $48 \%$ in 2011 ( $P=.0037)$. This trend was observed in clipping patients $(33-65 \%, P<.0001)$ but not coiling patients $(43-45 \%, P=.62)$.

CONCLUSIONS: For the pretreatment evaluation of ruptured aneurysms, the use of CT angiography increased from 2006-2011 without a corresponding decrease in the use of DSA. These results raise the question of potential redundancy without added clinical value of the second test.

$T^{1}$ he standard examination to evaluate for a source of subarachnoid hemorrhage for decades has been conventional angiography, which has now evolved into digital subtraction angiography. This is reflected in the American Heart Association/ American Stroke Association guidelines for the management of aneurysmal SAH, which strongly recommend DSA in their class I recommendations. ${ }^{1}$ In recent years, however, surgery without DSA on the basis of CT angiography has been advocated. ${ }^{2-5} \mathrm{~A}$ recent meta-analysis concluded that CT angiography can be used as a primary examination tool in the diagnostic work-up of patients with $\mathrm{SAH},{ }^{6}$ but that conclusion has been questioned because of weaknesses of CT angiography in the detection of small aneurysms and aneurysms adjacent to the skull base. ${ }^{7-9}$ Whether the imaging method used is DSA or CT angiography, the imaging

Received September 21, 2012; accepted after revision December 6.

From the Departments of Radiology (J.S.M., D.F.K., G.L., H.J.C.) and Neurosurgery (D.F.K., G.L., H.J.C.), Mayo Clinic, Rochester, Minnesota.

Please address correspondence to Jennifer S. McDonald, PhD, Department of Radiology, Mayo Clinic, 200 First St SW, Rochester, MN 55905; e-mail:

mcdonald.jennifer@mayo.edu

http://dx.doi.org/10.3174/ajnr.A3478 must identify the source of bleeding, most commonly an aneurysm. We evaluated a large, multihospital data base to determine how practice is evolving with regard to the use of CT angiography and DSA in patients with ruptured cerebral aneurysm.

\section{MATERIALS AND METHODS}

\section{Study Population and Data Retrieval}

The Perspective data base is a voluntary, fee-supported collection of data developed by Premier, Inc, to assess quality and resource use. ${ }^{10}$ As of 2011, the Perspective data base contained information from more than 600 US hospitals and consisted of approximately $15 \%$ of hospitalizations nationwide. This data base contains detailed hospitalization information, including patient and hospital demographics, diagnoses, and all billed items including procedures and diagnostic tests administered in relation to the day of admission. Because the Perspective data base consists of only de-identified patient and hospital data, our institutional review board deemed this study exempt from review and patient consent.

Patients who presented with subarachnoid hemorrhage (ICD9-CM diagnostic code 430) from 2006-2011 were identified. Pa- 
Patient and hospital demographics

\begin{tabular}{lcc}
\hline & Clipping & Coiling \\
\hline $\begin{array}{l}\text { Patient } \\
\text { Number of patients }\end{array}$ & 1022 & \\
$\begin{array}{l}\text { Age, } y, \text { median (interquartile } \\
\text { range) }\end{array}$ & $53(45-61)$ & $55(46-65)$ \\
$\quad \begin{array}{l}\text { Female sex, } n(\%) \\
\text { Hospital }\end{array}$ & $667(65 \%)$ & $2800(71 \%)$ \\
$\quad$ Region, $n(\%)$ & & \\
$\quad$ Midwest & & \\
$\quad$ South & $156(15 \%)$ & $447(11 \%)$ \\
$\quad$ Northeast & $436(43 \%)$ & $1666(42 \%)$ \\
$\quad$ West & $273(27 \%)$ & $1290(33 \%)$ \\
$\quad$ Median number of beds (range) & $623(447-725)$ & $623(14 \%)$ \\
$\quad$ Urban location, $n$ (versus rural) & $1011(99 \%)$ & $3857(98 \%)$ \\
$\quad$ Teaching, $n$ (versus nonteaching) & $699(68 \%)$ & $2684(68 \%)$ \\
\hline
\end{tabular}

tients were included if they underwent aneurysmal clipping (ICD-9 procedural code 39.51, "clipping of aneurysm") or coiling (ICD-9 procedural codes 39.52, ["other repair of aneurysm"], 39.72 [“endovascular repair of occlusion of head and neck vessels"], 39.75 [“endovascular embolization or occlusion of vessel[s] of head or neck by using bare coils"], 39.76 ["endovascular embolization or occlusion of vessel[s] of head or neck by using bioactive coils"], and 39.79 ["other endovascular repair [of aneurysm] of other vessels"]) during hospitalization. Patient billing information was used to confirm clipping patients by the presence of an aneurysm clip and to confirm coiling patients by the presence of endovascular coil(s).

Hospitals were defined as clipping or coiling centers if they performed clipping or coiling procedures at a rate of at least 5:1 over the 2006-2011 timeframe compared with coiling or clipping, respectively. Hospitals that performed both clipping and coiling at $<5: 1$ ratio were defined as performing both procedures.

Billing information was used to retrieve use of DSA or CT angiography during hospitalization. Day of DSA or CT angiography in relation to the day of admission was retrieved. Patients were included if they had DSA of the carotid or vertebral arteries or CT angiography of the head before clipping or coiling, defined as the day before or day of the procedure. The use of CT angiography and DSA at any point after clipping or coiling to discharge was also retrieved.

\section{Statistics}

Data were extracted from the Perspective data base by use of SAS (SAS, version 9.3; SAS Institute, Cary, North Carolina) and analyzed by use of JMP (version 9, SAS Institute). Continuous results are presented as median and interquartile range to account for nonparametric data distributions. Categoric results are presented as percentages. Differences in imaging use in 2006 and 2011 were compared by use of Fisher exact test.

\section{RESULTS}

\section{Study Population}

A total of 4972 patients were identified, of which 1022 were clipping cases and 3950 were coiling cases. Patient demographic characteristics are shown in the Table. A total of 116 hospitals were represented in the 2006-2011 timeframe. Most hospitals were urban, and more than half were teaching hospitals.

\section{Pretreatment Imaging}

Trends in pretreatment imaging from 2006-2011 are shown in Fig 1. A wide variation in use of imaging was observed. Overall,
$65 \%$ (3216/4972) of patients with SAH underwent DSA only, 6\% (301/4972) underwent CT angiography only, and 29\% (1455/ 4972) underwent both DSA and CT angiography before clipping or coiling. The use of CT angiography was almost twice as high in hospitals that predominantly performed clipping $(61 \%, 150 / 247)$ compared with hospitals that predominantly performed coiling (34\%, 765/2251, $P<.0001)$ or hospitals that performed both procedures $(34 \%, 841 / 2474, P<.0001)$. For clipping patients, $56 \%$ (570/1022) underwent DSA only, 20\% (210/1022) underwent CT angiography only, and 24\% (242/1022) underwent both DSA and CT angiography. For coiling patients, 67\% (2646/3950) underwent DSA alone and 33\% (1304/3950) underwent both DSA and CT angiography.

The use of pretreatment CT angiography and DSA varied, depending on the hospital. Teaching hospitals had significantly higher rates for the use of CT angiography (40\% [1362/3383] versus $25 \%$ [391/1589], $P<.0001)$ and lower rates for the use of DSA (93\% [3145/3383] versus 96\% [1521/1589], $P<.0001)$ compared with nonteaching hospitals. High-volume centers $(\geq 20$ cases/year) had higher rates for the use of DSA (96\% [2844/2962] versus $91 \%$ [1822/2010]), $P<.0001)$ and lower rates for the use of CT angiography (33\% [990/2962] versus 38\% [763/2010], $P=$ .0011) compared with low-volume centers. Rates for the use of DSA were higher in the Northeast (95\% [1477/1563]), South (94\% [1977/2102]), and West (92\% [643/704]) hospitals compared with Midwest hospitals (89\% [569/603]). Rates for the use of CT angiography were higher in Northeast hospitals (43\% [672/ $1563])$, compared with South (32\% [676/2102]), West (33\% [220/704]), and Midwest (31\% [185/603]) hospitals.

The percentage of patients with SAH who underwent pretreatment CT angiography significantly increased from 20\% (157/771) in 2006 to $44 \%(377 / 852)$ in $2011(P<.0001)$, whereas the percentage of patients who underwent DSA remained unchanged from $96 \%$ (734/771) to $94 \%(800 / 852)(P=.28)$. The fraction of patients who underwent pretreatment CT angiography increased from 20062011 in patients undergoing coiling (17\% [104/604] to 42\% [296/ $702], P<.0001)$ and in patients undergoing clipping (32\% [53/167] to $54 \%[81 / 150], P<.0001)$. The fraction of patients who underwent DSA decreased from $84 \%(140 / 167)$ to $77 \%(115 / 150)(P=.12)$ for patients treated with clipping. All coiling patients underwent DSA.

\section{Posttreatment Imaging}

Trends in posttreatment imaging are shown in Fig 2. Overall, 43\% $(2162 / 4972)$ of patients who received treatment for SAH underwent imaging after clipping or coiling, with 22\% (1096/4972) receiving DSA only, 12\% (575/4972) receiving CT angiography only, and 10\% (491/4972) receiving both DSA and CT angiography. Rates for the use of posttreatment imaging were higher in hospitals that predominantly performed coiling (52\% [1180/ 2251]) compared with hospitals that predominantly performed clipping (34\% [85/247], $P<.0001)$ or hospitals that performed both procedures (36\%, [897/2474], $P<.0001)$.

Rates for the use of posttreatment CT angiography and DSA varied, depending on the hospital. Teaching hospitals had a similar rate for the use of DSA (31\% [1057/3383] versus 33\% [530/ 1589], $P=.14$ ) but a higher rate for the use of CT angiography (22\% [761/3383] versus 19\% [305/1589], $P=.0085)$ compared 

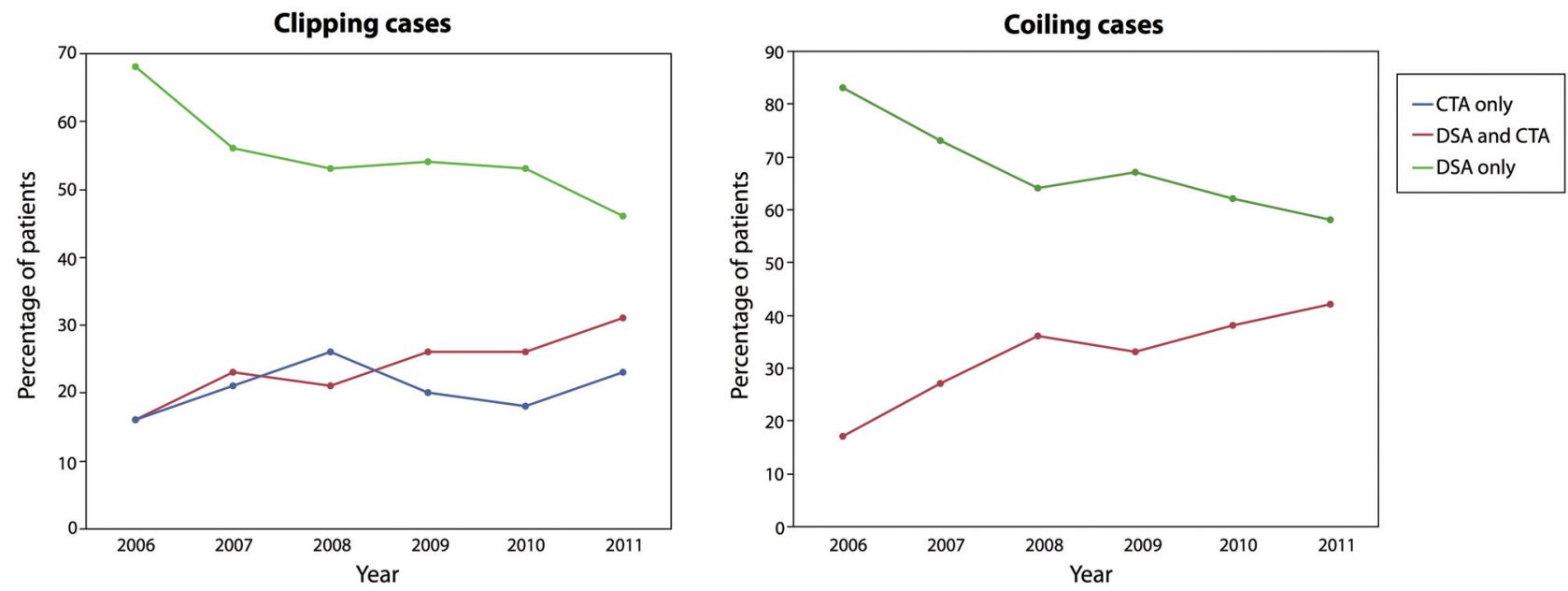

FIG 1. Trends in CT angiography and DSA use before clipping or coiling of ruptured aneurysms. Percentages of patients who underwent CT angiography only (blue line), DSA only (green line), or both CT angiography and DSA (red line) before clipping (left graph) or coiling (right graph) procedures from 2006-2011 are shown.
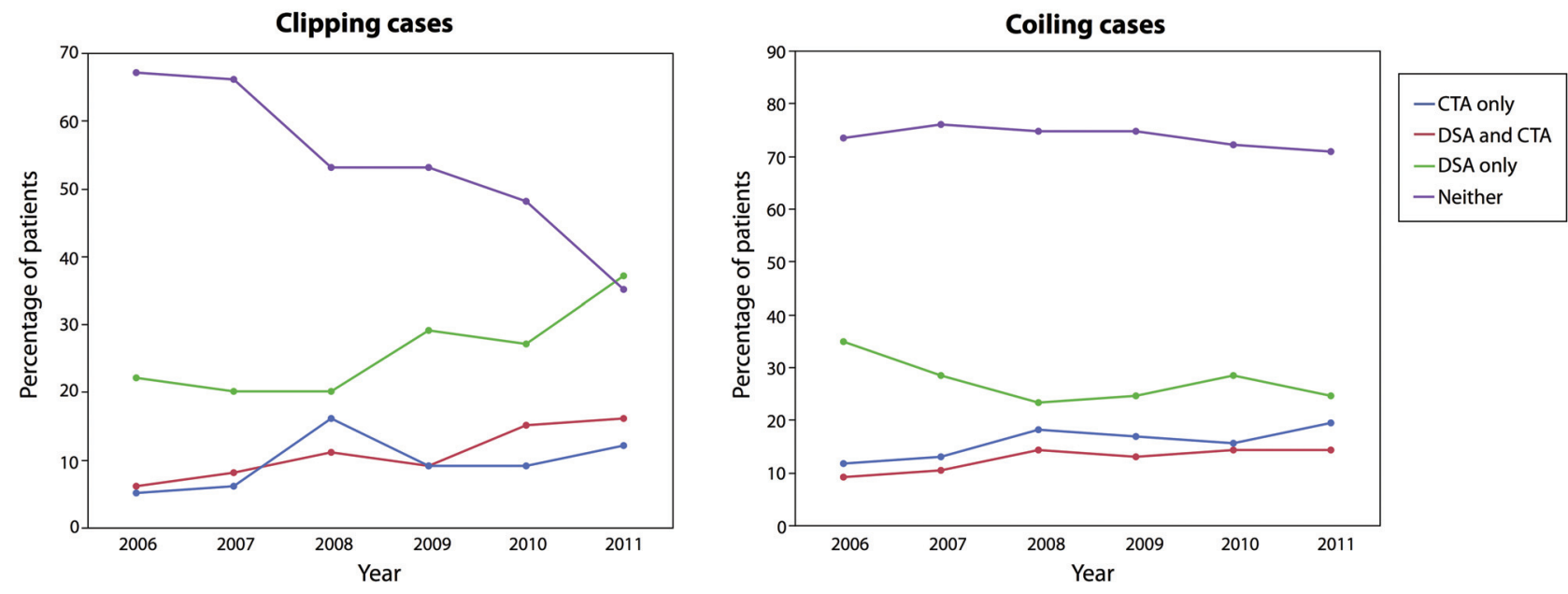

FIG 2. Trends in CT angiography and DSA use after clipping or coiling of ruptured aneurysms. Percentages of patients who underwent CT angiography only (blue line), DSA only (green line), both CT angiography and DSA (red line), or neither (purple line) after clipping (left graph) or coiling (right graph) procedures from 2006-2011 are shown.

with nonteaching hospitals. High-volume centers ( $\geq 20$ cases/ year) also had similar rates for the use of DSA (31\% [920/2962] versus 33\% [667/2010], $P=.12$ ) but higher rates for the use of CT angiography (23\% [684/2962] versus 19\% [382/2010], $P=.0011)$ compared with low-volume centers. Rates for the use of DSA were higher in West hospitals (40\% [282/704]) compared with South (29\% [609/2102]), Northeast (31\% [491/1563]), and Midwest (34\% [205/603]) hospitals. Rates for the use of CT angiography were higher in Midwest hospitals (30\% [180/603]) compared with Northeast (22\% [348/1563]), South (19\% [391/2102]), and West (21\% [147/704]) hospitals.

There was a significant increase in the percentage of patients who underwent posttreatment imaging from 41\% (317/771) in 2006 to $48 \%(412 / 852)$ in $2011(P=.0037)$. This increase was attributable to the increase in the rate for the use of CT angiography from $15 \%$ $(117 / 771)$ to $26 \%(221 / 852, P<.0001)$. Patients treated with clipping were increasingly subjected to posttreatment imaging from 2006-2011. The fraction of clipping patients who underwent posttreatment imaging significantly increased from 33\% (55/167) in 2006 to $65 \%(97 / 150)$ in $2011(P<.0001)$, with increases in the rate for the use DSA alone (from 22\% [36/167] to 37\% [55/150], $P=.0041$ ), CT angiography alone (from 5\% [9/167] to $12 \%$ [18/150], $P=.0436$ ), and both DSA and CT angiography (from 6\% [10/167] to 16\% [24/ $150], P=.0058)$. Conversely, the fraction of coiling patients who underwent posttreatment imaging increased minimally from $43 \%$ [262/604] to $45 \%$ [315/702], $P=.62)$.

\section{DISCUSSION}

Our study demonstrates that most patients continue to undergo DSA before clipping or coiling treatment of a ruptured aneurysm, but $20 \%$ of patients treated with clipping were imaged with CT angiography only. Rates for the use of CT angiography were higher in teaching and high-volume medical centers compared with nonteaching and low-volume medical centers. We also found higher rates for the use of CT angiography at centers that predominantly perform clipping compared with centers that predominantly perform coiling. DSA has historically been used to diagnose aneurysms in the setting of SAH, and the use of CT angiography in this context is a recent 
phenomenon. Rates for the use of DSA did not decrease substantially from 2006-2011, indicating that CT angiography tends to be used in most cases as a study in addition to DSA rather than as a replacement of DSA. Performance of both CT angiography and DSA before treatment of an aneurysm can increase the cost of treatment and the risk of contrast-induced nephropathy relative to the use of either technique alone, with unclear clinical benefit in many cases.

Although every patient who is treated with coiling undergoes DSA of the aneurysm being treated, it may not be a complete, 4-vessel DSA if a CT angiogram is deemed adequate for evaluation of the remaining blood vessels. We are not able to determine the degree of completeness of the DSA in this study; therefore we cannot ascertain how often CT angiography led to an abbreviated DSA.

Because of the noninvasive nature of $\mathrm{CT}$ angiography relative to DSA, an argument might be made that the use of CT angiography might be safer than the use of DSA. Through the use of the data base in this study, we cannot accurately assess complications of cerebral angiography to determine whether avoiding DSA by performing CT angiography resulted in better patient outcomes. Because the risk of permanent sequelae from DSA performed in patients with SAH is very low, ${ }^{11}$ an improvement in safety resulting from avoidance of DSA would be difficult to confirm. Even at centers that generally use DSA as the primary imaging, CT angiography might occasionally be used in the situation of an aneurysm causing parenchymal hematoma requiring urgent surgery, which occurs most commonly with middle cerebral artery aneurysms.

Patients treated with clipping were increasingly subjected to posttreatment CT angiography and/or DSA imaging from 2006-2011, whereas posttreatment imaging of patients undergoing coiling remained fairly constant. The use of more imaging after clipping compared with coiling might be at least partly because all patients undergoing coiling receive angiographic assessment at the end of the procedure, but patients undergoing clipping often do not receive angiographic assessment. We are not able to identify intraoperative DSA in the clipping cohort. This increasing trend may suggest an increasing desire by surgeons to get confirmation of adequate clipping with DSA. CT angiography and DSA in the days after clipping or coiling might be performed to evaluate the treatment result or to evaluate vasospasm. Vasospasm and delayed ischemic deficits occur less frequently in patients treated with coiling relative to those treated with clipping, ${ }^{12}$ which might contribute to less DSA and CT angiography after the aneurysm is treated by coiling.

This study has several limitations. First, this study is retrospective, and the decision of whether to perform CT angiography or DSA is ultimately made by the provider. Second, indications for imaging are not available in the Perspective data base; therefore, the clinical decision-making process for each hospitalization and the reasons that providers chose CT angiography, DSA, or both modalities are unknown. The implications of our findings may be different for pretreatment and posttreatment imaging because they have different indications. Third, the Perspective data base only captures imaging that was performed during hospitalization. Imaging that may have been performed immediately before or after hospitalization would therefore have been missed. Fourth, the data base is not a random sampling of hospitals, and therefore results may not be representative of all hospitals or hospitalizations. Fifth, some coding inaccuracies undoubtedly occur that can affect the retrospective evaluation of an administrative data base. Such coding inaccuracies are unlikely to lead to misrepresentation of overall trends in the use of imaging. Sixth, whereas our study shows an increasing trend in the use of CT angiography, the level of use of DSA and CT angiography considered optimal or appropriate is unknown. Finally, the findings of our study are only applicable to ruptured aneurysms. The use of DSA and CT angiography may be different with unruptured aneurysms or SAH from other causes.

\section{CONCLUSIONS}

For the pretreatment evaluation of ruptured aneurysms, the use of CT angiography increased from 2006-2011, but without a corresponding decrease in the use of DSA. The increased use of CT angiography without a corresponding decrease in DSA use raises the question of potential redundancy in the diagnostic tests without added clinical value of the second test.

Disclosures: Jennifer McDonald_UNRELATED: Grants/Grants Pending: GE Healthcare. * David Kallmes-UNRELATED: Consultancy: ev3,* Medtronic, ${ }^{*}$ Codman; ${ }^{*}$ Grants/Grants Pending: MicroVention,* ev3,* Sequent,* Benvenue Medical,* Micrus, ${ }^{*}$ Codman;* Royalties: UVA Patent Foundation; Payment for Development of Educational Presentations: ev3;* Travel/Accommodations/Meeting Expenses Unrelated to Activities Listed: MicroVention. * Giuseppe Lanzino-UNRELATED: Consultancy: ev3/Covidien;* Travel/Accommodations/Meeting Expenses Unrelated to Activities Listed: ev3/Covidien, ${ }^{*}$ Comments: FDA panel. Harry Cloft—UNRELATED: Grants/Grants Pending: Cordis Endovascular.* ("Money paid to institution.)

\section{REFERENCES}

1. Bederson JB, Connolly ES Jr, Batjer HH, et al. Guidelines for the management of aneurysmal subarachnoid hemorrhage: a statement for healthcare professionals from a special writing group of the stroke council, American Heart Association. Stroke 2009;40:994-1025

2. Agid R, Lee SK, Willinsky RA, et al. Acute subarachnoid hemorrhage: using 64-slice multidetector CT angiography to "triage" patients' treatment. Neuroradiology 2006;48:787-94

3. Agid R, Willinsky RA, Farb RI, et al. Life at the end of the tunnel: why emergent CT angiography should be done for patients with acute subarachnoid hemorrhage. AJNR Am J Neuroradiol 2008;29:e45

4. Fox AJ, Symons SP, Aviv RI. CT angiography is state-of-the-art first vascular imaging for subarachnoid hemorrhage. AJNR Am J Neuroradiol 2008;29:e41-42

5. Gonzalez-Darder JM, Pesudo-Martinez JV, Feliu-Tatay RA. Microsurgical management of cerebral aneurysms based in CT angiography with three-dimensional reconstruction (3D-CT angiography) and without preoperative cerebral angiography. Acta Neurochirurgica 2001;143:673-79

6. Westerlaan HE, van Dijk JM, Jansen-van der Weide MC, et al. Intracranial aneurysms in patients with subarachnoid hemorrhage: $C T$ angiography as a primary examination tool for diagnosissystematic review and meta-analysis. Radiology 2011;258:134-45

7. Jayaraman MV, Haas RA, Do HM, et al. Should CT angiography be routinely used in patients suspected of having aneurysmal subarachnoid hemorrhage? No! Radiology 2010;254:314-15

8. Kallmes DF, Layton K, Marx WF, et al. Death by nondiagnosis: why emergent $\mathrm{CT}$ angiography should not be done for patients with subarachnoid hemorrhage. AJNR Am J Neuroradiol 2007;28:1837-38

9. Moran CJ. Aneurysmal subarachnoid hemorrhage: DSA versus CT angiography-is the answer available? Radiology 2011;258:15-17

10. Premier Perspective Database. Charlotte, North Carolina: Premier Research Services. 2012

11. Cloft HJ, Joseph GJ, Dion JE. Risk of cerebral angiography in patients with subarachnoid hemorrhage, cerebral aneurysm, and arteriovenous malformation: a meta-analysis. Stroke 1999;30:317-20

12. Dumont AS, Crowley RW, Monteith SJ, et al. Endovascular treatment or neurosurgical clipping of ruptured intracranial aneurysms: effect on angiographic vasospasm, delayed ischemic neurological deficit, cerebral infarction, and clinical outcome. Stroke 2010;41:2519-24 